\title{
DIABETES
}

\section{Efficacy of dapagliflozin associated with renal function}

A treatment option for patients with type 2 diabetes mellitus (T2DM) is inhibition of the sodium-glucose cotransporter 2 (SGLT2). Using this therapy, improvement in glycaemic control is dependent on the ability of the kidney to filter glucose. Therefore, it was reasonable to question whether patients with moderate renal impairment will have the same glycaemic responses to SGLT2 inhibitors as do those with normal or mildly impaired renal function. The answer has been revealed in results from a phase II-III, randomized, trial led by Donald Kohan.

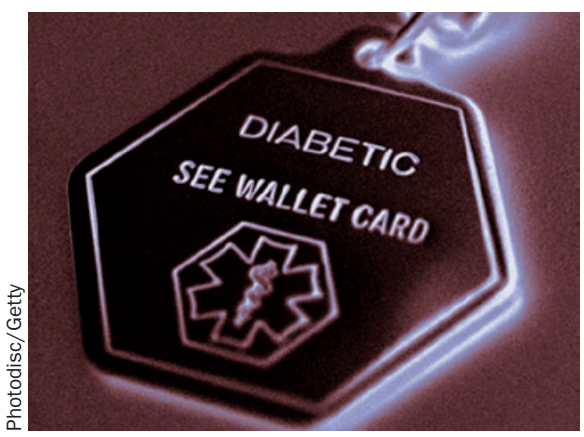

"Previous studies show that [the SGLT2 inhibitor] dapagliflozin reduces hyperglycaemia in patients with diabetes and normal or mildly impaired renal function," explains Kohan. "However, the efficacy of any SGLT2 inhibitor in reducing hyperglycaemia in stage 3 chronic kidney disease (CKD) had not been investigated." In this trial, 252 patients with T2DM and moderate renal impairment were randomly assigned to dapagliflozin or placebo and followed for up to 104 weeks.

The primary end point-mean change in HbA1c levels at 24 weeks-was not significantly different when comparing the two groups. This lack of an effect of dapagliflozin on hyperglycaemia in the study population is in contrast to its beneficial effects in patients with T2DM and more-normal renal function. However, the difference in efficacy would have been predicted based on the mechanism of action.

Some positives can be taken from the trial, however, as Kohan outlines, "body weight, blood pressure and hyperkalaemic episodes were lower in dapagliflozintreated, as compared to placebo-treated, patients with stage $3 \mathrm{CKD}$. Creatinine clearance and estimated glomerular filtration rate (eGFR) were mildly decreased in the dapagliflozin groups after 1 week of treatment, but then stabilized, whereas eGFR on placebo gradually decreased." Taken together, it seems that despite the lack of efficacy in glycaemic control, dapagliflozin therapy does have some benefits in this patient group.

The researchers believe that based on these data "further study regarding the appropriate use of dapagliflozin, and of SGLT2 inhibitors in general, in patients with stage 3 CKD should be carried out."

Rebecca Kirk

Original article Kohan, D. E. et al. Long-term study of patients with type 2 diabetes and moderate renal impairment shows that dapagliflozin reduces weight and blood pressure but does not improve glycemic control. Kidney Int. doi:10.1038/ki.2013.356 